\title{
AN ALGORITHM FOR REAL AND COMPLEX RATIONAL MINIMAX APPROXIMATION*
}

\author{
YUJI NAKATSUKASA ${ }^{\dagger}$ AND LLOYD N. TREFETHEN ${ }^{\dagger}$
}

\begin{abstract}
Rational minimax approximation of real functions on real intervals is an established topic, but when it comes to complex functions or domains, there appear to be no algorithms currently in use. Such a method is introduced here, the AAA-Lawson algorithm, available in Chebfun. The new algorithm solves a wide range of problems on arbitrary domains by a procedure consisting of two steps. First, the standard AAA algorithm is run to obtain a near-best approximation and a set of support points for a barycentric representation of the rational approximant. Then a "Lawson phase" of iteratively reweighted least-squares adjustment of the barycentric coefficients is carried out to improve the approximation to minimax.
\end{abstract}

Key words. rational approximation, barycentric formula, AAA algorithm, AAA-Lawson algorithm, iteratively reweighted least-squares

AMS subject classifications. 41A20, 65D15

DOI. $10.1137 / 19 \mathrm{M} 1281897$

1. Introduction. Rational minimax approximation - the optimal approximation of a function $f$ by a rational function $r$ of a given degree on a given domain in the supremum norm - is an old idea. For real approximation on a real interval, best approximations exist and are unique and are characterized by an equioscillation condition. Algorithms appeared beginning in the 1960s [50, 89, 90], and the problem became important for applications in the 1970s with the development of digital signal processing [58]. A powerful implementation is available in the minimax command in Chebfun [19, 23]. For complex functions or domains, however, the situation is very different. The theory developed by Walsh in the 1930s shows that existence and especially uniqueness may fail $[86,87]$, and as for algorithms, there is not much available apart from a pair of methods introduced by Ellacott and Williams (EW) (1976) and Istace and Thiran (IT) (1993) based on earlier work by Osborne and Watson [60], which, as far as we are aware, are not in use today [22, 40] (see section 7). This is a striking gap, since rational approximations are of growing importance in computational complex analysis (Figure 4.3) [29, 83], systems theory and model order reduction (Figure 4.6) $[2,3,7,12]$, low-rank data compression (Figures 4.6 and 4.7) $[6,46]$, electronic structure calculation (Figure 5.2) [48, 53], and solution of partial differential equations (Figure 5.4) [15, 20,30,31].

The aim of this paper is to introduce a new algorithm for complex rational minimax approximation together with a software implementation. Our "AAA-Lawson" algorithm combines the rational barycentric AAA algorithm of [54] with an iteratively reweighted least-squares (IRLS) iteration inspired by Lawson's algorithm [44] but in a nonlinear barycentric context. It works on discrete domains, typically containing hundreds or thousands of points to approximate a continuum, which may take all kinds of forms, including Jordan regions, unions of Jordan regions, regions with holes, intervals, unbounded domains, clouds of random points, and more. Being based on a

*Submitted to the journal's Methods and Algorithms for Scientific Computing section August 6, 2019; accepted for publication (in revised form) July 8, 2020; published electronically October 13, 2020.

https://doi.org/10.1137/19M1281897

${ }^{\dagger}$ Mathematical Institute, University of Oxford, Oxford, OX2 6GG, UK (nakatsukasa@maths.ox. ac.uk, trefethen@maths.ox.ac.uk). 
barycentric rational representation with greedy selection of support points, it inherits the exceptional numerical stability of AAA and is able to handle even very difficult cases with exponentially clustered poles. Experiments show that for a wide range of problems, the method converges quickly to an approximation whose error is close to the minimax value.

The version of the AAA-Lawson algorithm described here was introduced in the aaa command of Chebfun in August, 2019, and we hope its easy availability may open up a new era of exploration of complex rational minimax approximation. For example, Ellacott and Williams list minimax errors for 29 different approximation problems in Tables 1 and 2 of their paper, each given to 3 digits of accuracy [22]. With Chebfun aaa, all of these problems can be solved in a total time of less than 2 seconds on a desktop computer, along with the three additional problems left blank in their tables. (For hardware details, see section 4.) Twelve of the EW numbers turn out to be correct to all three digits, with the rest having small anomalies mainly associated with discretization of a continuum by too few points. We shall discuss comparisons with the EW and IT algorithms in section 7 .

For complex polynomial approximation, more computational possibilities are available than in the rational case, including [5, 24, 28, 43, 57, 75]. The "complex Remez algorithm" of Tang is particularly appealing [75]. Similarly, there are a number of non-minimax complex rational approximation algorithms, including vector fitting [33], RKFIT [8], the Loewner framework [3], IRKA [32], and AGH [1], as well as the AAA algorithm that is our own starting point [54]. Most of these methods apply to vector or matrix as well as scalar approximation problems, whereas the AAA-Lawson method has only been developed so far for scalars. To extend it, one could perhaps adapt some of the methods proposed for AAA in [47].

The possibility of an AAA-Lawson algorithm was first mentioned in the original AAA paper [54], and it was developed further for part of the initialization process for the Chebfun minimax command [23]. However, in these projects the power of AAA-Lawson for general minimax approximation did not become fully apparent for a number of reasons. One was that AAA approximations are usually computed with the degree not specified but adaptively chosen to get down to nearly machine precision, and in this setting, AAA-Lawson will usually fail (it is trying to improve a result that is already near the limit of precision). Another is that much of our attention was on real intervals, where both AAA and AAA-Lawson are least robust. A third was that we did not fully appreciate the crucial importance of choosing approximation grids exponentially clustered near corners and other singular points, where poles of rational approximations will be exponentially clustered. Finally, in those experiments we were not including the support points themselves in the matrix associated with the IRLS problem (see (3.5) and the discussion preceding it), an omission that led to failure in some cases.

We close this introduction with Figure 1.1, illustrating the behavior of the algorithm in a typical problem (the first example of section 4). The first, the AAA phase, rapidly finds a near-minimax approximation, and this is improved to minimax in the second, the Lawson phase. This figure vividly raises a fundamental question. Is it worthwhile to complicate an algorithm significantly, and slow it down by a factor of 2 or more, to achieve just one more digit of accuracy in an approximation? From a practical point of view, we believe the answer is sometimes yes and sometimes no. Optimal approximations have long been regarded as important in signal processing applications, for example, but in other larger-scale and less-embedded contexts, they may be less so. However, there is also the theoretical point of view. Minimax approx- 

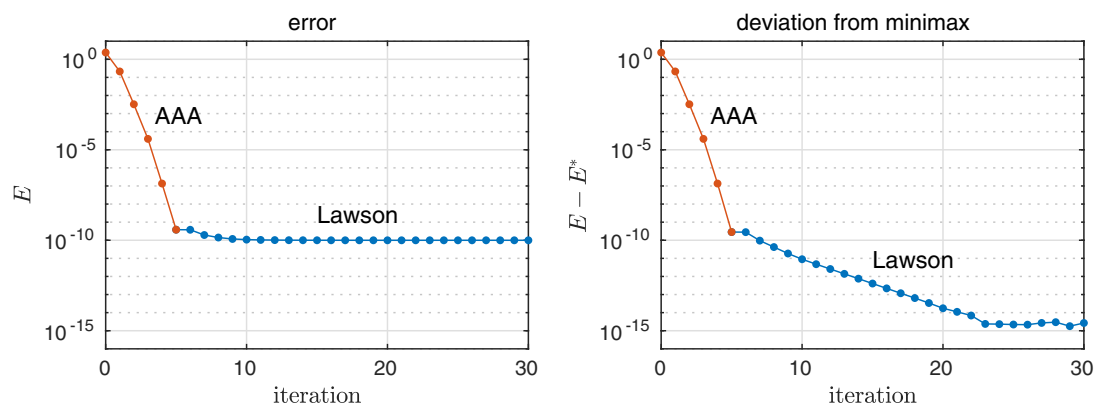

FIG. 1.1. The two phases of the AAA-Lawson algorithm, illustrated here for degree 5 approximation of $e^{z}$ on the unit disk. The AAA phase achieves rapid convergence to a near-minimax approximant. This is then improved to minimax by a linearly convergent Lawson iteration.

imations, with their distinctive error curves that the rest of the figures of this paper will illustrate, have exercised a fascination since the days of Chebyshev, and they have been the subject of a great deal of study by approximation theorists. An appealing aspect of the AAA-Lawson algorithm is that it will facilitate new explorations in this area.

2. Existence, uniqueness, characterization, and convergence. A rational function is a function of a complex variable that can be written in the form $r(z)=$ $p(z) / q(z)$, where $p$ and $q$ are polynomials. We say that $r$ is of type $(m, n)$ for some $m, n \geq 0$ if it can be represented with $p$ of degree at most $m$ and $q$ of degree at most $n$. If $m=n$, the setting of this paper, we also say that $r$ is of degree $n$, and we denote by $R_{n}$ the set of rational functions of degree $n$. A rational function is a map from $\mathbf{C}^{+}$to $\mathbf{C}^{+}$, where $\mathbf{C}^{+}$is the extended complex plane $\mathbf{C} \cup\{\infty\}$, and if $r \in R_{n}$ is not a constant, then it takes each value in $\mathbf{C}^{+}$at most $n$ times, counted with multiplicity. It is the function that is the fundamental object, not any particular representation of it, and if a representation has isolated points corresponding to quotients $0 / 0$ or $\infty / \infty$, then we define the values there by limits from neighboring points.

Let $Z \subseteq \mathbf{C}$ be nonempty, let $\|\cdot\|$ be the supremum norm on $Z$, and let $f$ be a complex continuous function (not necessarily analytic) defined on $Z$. Our approximation problem is to find rational functions $r$ such that $\|f-r\|$ is small. If $r^{*} \in R_{n}$ satisfies $\left\|f-r^{*}\right\|=E^{*}:=\inf _{r \in R_{n}}\|f-r\|$, then $r$ is a best or minimax approximation to $f$ of degree $n$. Even if there is no minimax approximation, we will speak of the minimax error $E^{*}$, which may be any number in the range $[0, \infty]$.

Polynomial best approximations of a given degree $n$ always exist, and if $Z$ is a compact set with at least $n+1$ points, then they are unique [77]. They can be characterized by a condition due to Kolmogorov [42, 57, 71, 76], and if $Z$ is a closed real interval of positive length and $f$ is real on $Z$, then there is a simpler and more famous characterization by equioscillation of the error $(p-f)(x)$ between $\geq n+2$ alternating extreme points. In this case, $E^{*}$ decreases exponentially as $n \rightarrow \infty$ if and only if $f$ is analytic on $Z$ [81, Chapter 8], and the same result generalizes to any compact set $Z \subseteq \mathbf{C}$ [87].

The theory of complex rational best approximation, which begins with a 1931 paper by Walsh [86, 87], proves more problematic. First of all, best approximations need not exist. For example, there is no degree 1 best approximation to data values $a, b, b$ with $a \neq b$ on any set $Z$ with three distinct points, for in such a case we have $E^{*}=0$ but $\|f-r\|>0$ for any choice of $r$, since a nonconstant function $r \in R_{1}$ 
can only take each value once. However, Theorem 3 of [86] asserts that existence is assured if $E^{*}<\infty$ and $Z$ has no isolated points.

Concerning uniqueness, there is one main positive result: if $Z$ is a closed real interval of positive length and $f$ is real, then a best real rational approximation in $R_{n}$ exists and is unique and characterized by an error curve that equioscillates between sufficiently many extreme points. Without the restriction that $r$ is real, however, uniqueness is not assured [49, 66, 92]. For example, there are complex approximations to $|x|$ on $[-1,1]$ whose error is less than the value $1 / 2$ achieved by the best real approximation, from which it follows by the symmetry of complex conjugation that the complex best approximation cannot be unique (see Exercise 24.3 of [81]). Examples of nonuniqueness have also been investigated on the unit disk [36].

When it comes to characterization of rational best approximants, the Kolmogorov condition diminishes to a necessary condition for local optimality: for a candidate approximation to be locally optimal, it must be a minimal point with respect to certain local linear perturbations. Discussions can be found in a number of sources, including [34, 65, 71, 92], and we recommend in particular the papers [40, 76] by Istace and Thiran. Sufficient conditions, and conditions for global optimality, are mostly not available, though there are some results in [51,65]. We shall say more on these subjects in sections 6 and 7 .

These observations are daunting. However, although it is a fascinating mathematical challenge to elucidate the properties of best approximations, what matters for most applications is that we are able to compute good ones. An example is provided by the "lightning Laplace solver" paper [31], which presents far-reaching theorems about root-exponential convergence of rational approximations for solutions to Laplace problems with boundary singularities. The approximations are not minimax, but still they lead to a very fast Laplace solver.

A major focus of the theoretical literature of rational approximation is the problem of approximability, the determination of necessary and sufficient conditions to ensure that $E^{*} \rightarrow 0$ as $n \rightarrow \infty$ in approximation of a function $f$ on a set $Z \subseteq \mathbf{C}$. If $Z$ is compact with at most a finite number of holes and $f$ is analytic on $Z$, then exponential decrease of $E^{*}$ to 0 was established by Runge in 1885 [64], but what if $f$ is merely analytic in the interior? Here we cannot expect exponential convergence, but according to Vitushkin's theorem [26, 27, 93], the generalization to rational approximation of Mergelyan's theorem for polynomials, we still get $E^{*} \rightarrow 0$. And what if there are infinitely many holes? Vitushkin's theorem gives technical conditions for this case too. But such questions are a long way from most applications of rational approximation, where the whole point is to exploit circumstances in which $E^{*} \rightarrow 0$ very fast.

3. The AAA-Lawson algorithm. Let $n+1$ distinct support points $t_{0}, \ldots, t_{n} \in$ $\mathbf{C}$ be fixed for some $n \geq 0$, and let $\ell$ be the node polynomial

$$
\ell(z)=\prod_{k=0}^{n}\left(z-t_{k}\right)
$$

which is monic and of degree $n+1$. If $\alpha_{k}, \beta_{k} \in \mathbf{C}$ are arbitrary complex numbers, $0 \leq k \leq n$, with at least one $\beta_{k}$ being nonzero, then the quotient of partial fractions

$$
r(z)=\frac{n(z)}{d(z)}=\sum_{k=0}^{n} \frac{\alpha_{k}}{z-t_{k}} / \sum_{k=0}^{n} \frac{\beta_{k}}{z-t_{k}}
$$


is obviously a rational function of degree $2 n+1$, since this is true of any quotient of one rational function of type $(n, n+1)$ divided by another, so long as the latter is not identically zero. However, in this quotient the numerator and the denominator have the same poles, so $r$ is also of the smaller degree $n$. We can verify this algebraically by multiplying both the numerator and the denominator of (3.2) by $\ell$. The expression (3.2) is a barycentric representation for $r$ [9].

Conversely, regardless of the choice of the support points, every degree $n$ rational function $r$ can be written in the form (3.2). The following theorem is adapted from [54].

THEOREM 3.1 (rational barycentric representations). Let $t_{0}, \ldots, t_{n}$ be an arbitrary set of distinct complex numbers. As $\alpha_{0}, \ldots, \alpha_{n}$ and $\beta_{0}, \ldots, \beta_{n}$ range over all complex values, with at least one $\beta_{k}$ being nonzero, the functions (3.2) range over the set of all rational functions of degree $n$.

Proof. ${ }^{1}$ As just observed, any quotient (3.2) is a rational function $r$ of degree $n$. Conversely, suppose $r$ is a rational function of degree $n$, and write $r=p / q$, where $p$ and $q$ are polynomials of degree at most $n$. It is enough to show that coefficients $\left\{\alpha_{k}\right\}$ and $\left\{\beta_{k}\right\}$ exist such that $p=n \ell$ and $q=d \ell$ in (3.2). Now $d \ell$ is a linear combination with coefficients $\beta_{0}, \ldots, \beta_{n}$ of $n+1$ monic polynomials of degree $n$, which are linearly independent since they have different sets of roots. Thus $q$ can be written (uniquely) as $d \ell$, and similarly for $p=n \ell$.

This proof shows that there is a one-to-one correspondence between sets of coefficients $\left\{\alpha_{k}\right\}$ in a barycentric representation (3.2) and polynomials $p$ in a quotient representation $p / q$, and likewise for $\left\{\beta_{k}\right\}$ and $q$. Thus we see that the barycentric representation is unique to exactly the same degree as the quotient representation $p / q$ : unique up to a multiplicative constant if $r$ has degree $n$ but not $n-1$, with further nonuniqueness if $r$ is of degree $n-1$ or less.

Rational barycentric formulas with independent coefficients $\alpha_{k}$ and $\beta_{k}$ are not well known. Traditionally, barycentric formulas are used in "interpolatory mode," where function values $\left\{f_{k}\right\}$ are given and weights are chosen corresponding to $\alpha_{k} / \beta_{k}=f_{k}$ (and $\beta_{k} \neq 0$ ), yielding $r\left(t_{k}\right)=f_{k}$ for each $k[9,25,54,68]$. To work with arbitrary rational functions, however, with a complete decoupling of support points from approximation properties, one needs the "noninterpolatory" or "alpha-beta" mode (3.2). Ultimately, the $\alpha_{k}$ and $\beta_{k}$ are devoted to approximation and the $t_{k}$ to numerical stability.

The AAA-Lawson algorithm consists of two steps. We assume that a discrete domain $Z$ and a set of corresponding function values $F=f(Z)$ have been given, together with a degree $n$.

(I) Run the AAA algorithm to get a rational approximant $r^{(\mathrm{I})} \approx f$ of degree $n$ and a set of support points $t_{0}, \ldots, t_{n}$.

(II) Carry out a linearized barycentric Lawson iteration with the same support points $t_{0}, \ldots, t_{n}$ until a termination condition is reached.

Step (I) utilizes (3.2) in interpolatory mode, fixing $\alpha_{k}=f\left(t_{k}\right) \beta_{k}$ for each $k$. In outline, we begin with a support point $t_{0} \in Z$ where $|f(z)|$ attains its maximal value and the corresponding degree 0 rational approximant $r_{0}=f\left(t_{0}\right)$. Then, for $m=1,2, \ldots, n$, we take the next support point to be a point $t_{m} \in Z$ where $\mid f(z)-$

\footnotetext{
${ }^{1}$ An alternative proof can be based on the theory of partial fractions. The function $q / \ell$ is rational, with a zero at $\infty$ and a simple pole at each point $t_{k}$, or no pole at all if $q\left(t_{k}\right)=0$. This implies that $q / \ell$ can be written in the form $d$ of $(3.2)[38$, p. 553]. Similarly, $p / \ell$ can be written in the form $n$.
} 
$r_{m-1}(z) \mid$ attains its maximal value, and we define the degree $m$ rational function $r_{m}$ by minimizing $\|r d-n\|_{2}$, in the notation of (3.2), over all choices of $(m+1)$ vectors $\boldsymbol{\beta}=\left(\beta_{0}, \ldots, \beta_{m}\right)^{T}$ with $\|\boldsymbol{\beta}\|_{2}=1$. This calculation involves the singular value decomposition, and $\|r d-n\|_{2}$ is the discrete 2 -norm over the points of $Z$ that are not among the support points. Full details are presented in [54].

At the end of step (I), $\left\|f-r^{(\mathrm{I})}\right\|$ is typically within about an order of magnitude of the minimax error, but since $r^{(\mathrm{I})}$ interpolates the data at the support points, it cannot in general be the optimal approximant. It remains to describe step (II), which switches to noninterpolatory mode.

Let $Z=\left(z_{j}\right), 1 \leq j \leq M$, be the sample set, interpreted as a column vector, and let $F=\left(f_{j}\right), 1 \leq j \leq M$, be the corresponding vector of function values to be matched. Let $\alpha$ and $\beta$ be the coefficient vectors $\left(\alpha_{0}, \ldots, \alpha_{n}\right)^{T}$ and $\left(\beta_{0}, \ldots, \beta_{n}\right)^{T}$, with $\gamma$ defined as their concatenation $\gamma=[\alpha ; \beta]$. Our aim is to solve the minimax problem

$$
\min _{\gamma} \max _{j}\left|f_{j}-\sum_{k=0}^{n} \frac{\alpha_{k}}{z_{j}-t_{k}} / \sum_{k=0}^{n} \frac{\beta_{k}}{z_{j}-t_{k}}\right| .
$$

The barycentric Lawson idea is to achieve this by solving a sequence of iteratively reweighted least-squares (IRLS) problems based on the linearization of (3.3), now with $\alpha_{k}$ and $\beta_{k}$ independent,

$$
\min _{\gamma,\|\gamma\|_{2}=1} \sum_{j=1}^{M}{ }^{\prime} w_{j}\left(f_{j} \sum_{k=0}^{n} \frac{\beta_{k}}{z_{j}-t_{k}}-\sum_{k=0}^{n} \frac{\alpha_{k}}{z_{j}-t_{k}}\right)^{2},
$$

where at each step, $W=\left(w_{j}\right), 1 \leq j \leq M$, is a vector of weights $w_{j} \geq 0$. Note the prime symbol on the summation sign. This signifies that special treatment is applied at the $n+1$ sample points $z_{j}$ that coincide with a support point $t_{k}$ for some $k=k_{j}$. At these points the partial fractions in (3.4) will generally be infinite, and in the standard AAA algorithm, they are simply omitted from the least-squares problem since interpolation at these points is ensured by the barycentric formula. In the AAA-Lawson algorithm, however, $r$ will not necessarily interpolate $f$ at the support points, so it is necessary to include them explicitly in the approximation problem. (Experiments show that this becomes particularly important on coarser grids, where the omission of a single point may significantly affect the outcome. The examples of Figure 4.4 below are of this type.) The quantity that we include in (3.4) at these points is

$$
w_{j}\left(f_{j} \beta_{k_{j}}-\alpha_{k_{j}}\right)^{2}
$$

derived by dividing out the common factor approaching $\infty$ as $z \rightarrow t_{k_{j}}$, in the spirit of L'Hôpital's rule.

Equation (3.4) is a routine problem of numerical linear algebra, which can be written in matrix form as

$$
\min _{\gamma,\|\gamma\|_{2}=1}\left\|\operatorname{diag}\left(W^{1 / 2}\right)[C,-\operatorname{diag}(F) C] \gamma\right\|_{2},
$$

where $C$ is the Cauchy matrix with entries $c_{i j}=1 /\left(z_{j}-t_{k}\right)$ except in the $n+1$ special rows. This is a minimal singular value problem involving a matrix of size $M \times(2 n+2)$.

From one IRLS step to the next, $W$ is updated by the formula

$$
w_{j}^{(\text {new })}=w_{j}\left|e_{j}\right|
$$


where $e_{j}$ is the quantity inside absolute values in (3.3), i.e., the current nonlinear error at $z_{j}$. (For the $n+1$ special values of $j, e_{j}=f_{j}-\alpha_{k_{j}} / \beta_{k_{j}}$.) For convenience, and floating-point arithmetic, we then renormalize the weights at each step so that their maximum is 1 .

The IRLS idea originated with Lawson in 1961 [44] for linear minimax approximation, and has subsequently been analyzed and generalized by a number of authors beginning with Cline [14], Rice [62], and Rice and Usow [63]. Rice proved convergence at a linear rate for real approximation under natural assumptions [62], and Ellacott and Williams pointed out that the same proof extends to complex approximation [22]. IRLS algorithms have also taken on importance for other kinds of linear $L^{p}$ approximation, particularly the case $p=1$ of interest in data science [17, 59, 88]. Nonlinear generalizations of IRLS, though fewer, include [16, 70]. As far as we know, AAALawson is the first IRLS algorithm based on rational barycentric representations.

This completes our description of the core idea of the AAA-Lawson algorithm, but three questions remain. (i) How do we terminate the iteration? (ii) What can be proved about convergence? (iii) What measures can be taken to make convergence faster or more reliable in troublesome cases? Even for linear IRLS approximation, these are nontrivial matters, and the nonlinear case brings additional difficulties. Chebfun's current answer to (i) is that by default it takes 20 Lawson steps. This number is large enough so that the approximation is usually brought much closer to minimax, but small enough so that the additional expense is not too great. (In sections 4 and 5 , we shall see that the Lawson phase typically multiplies the overall computing time by a factor of $2-5$.) We would expect this very simple stopping condition to evolve as understanding of the algorithm deepens in the future. Questions (ii) and (iii) will be discussed in section 6 .

4. Numerical examples, complex. This section and the next are devoted to numerical examples. These were executed in Chebfun on an Intel i5-8500T CPU running at $2.10 \mathrm{GHz}$ (maximum speed $3.5 \mathrm{GHz}$ ), with $16 \mathrm{~GB}$ of RAM.

In this section, we present 14 examples of complex minimax approximations, grouped into pairs for convenience. Each example is represented by three images in the complex plane, the first showing the domain $Z$ and the second and third showing the error $r(Z)-f(Z)$ in AAA and AAA-Lawson approximation. All computations were done in Chebfun in the default mode, and computer timings are printed at the tops of the figures. The codes of this section and the next are available in the supplementary materials, linked from the main article webpage.

Analytic function on the unit disk. Figure 4.1 begins with the basic example of $e^{z}$ on the unit circle, discretized by 500 equispaced points. In 0.007 sec., AAA finds a near-best approximation for $n=5$ with error 3.83e-10. The black dots on the circle mark the six support points the algorithm selects. Continuing with the same support points but now in noninterpolatory "alpha-beta" mode, AAA-Lawson improves the approximation to close to minimax, with error 9.944364e-11. By the maximum modulus principle for analytic functions, these maximal errors on the circle are also the maximal errors over the whole disk. Note that the error curve appears to be a perfect circle (of winding number $2 n+1=11$, though this cannot be distinguished in the figure). This near-circularity effect was first identified in [78] and then investigated for polynomial approximation in [79] and rational approximation in [80]. The error curve cannot be exactly circular (this would imply that the function being approximated was rational), but as shown in [80], it comes spectacularly close, varying in radius for this example, we estimate via Theorem 6.3 of [80], by less than one part in $10^{12}$. This effect led to the theory of Carathéodory-Fejér (CF) approximation [37, 80, 81, 85], 

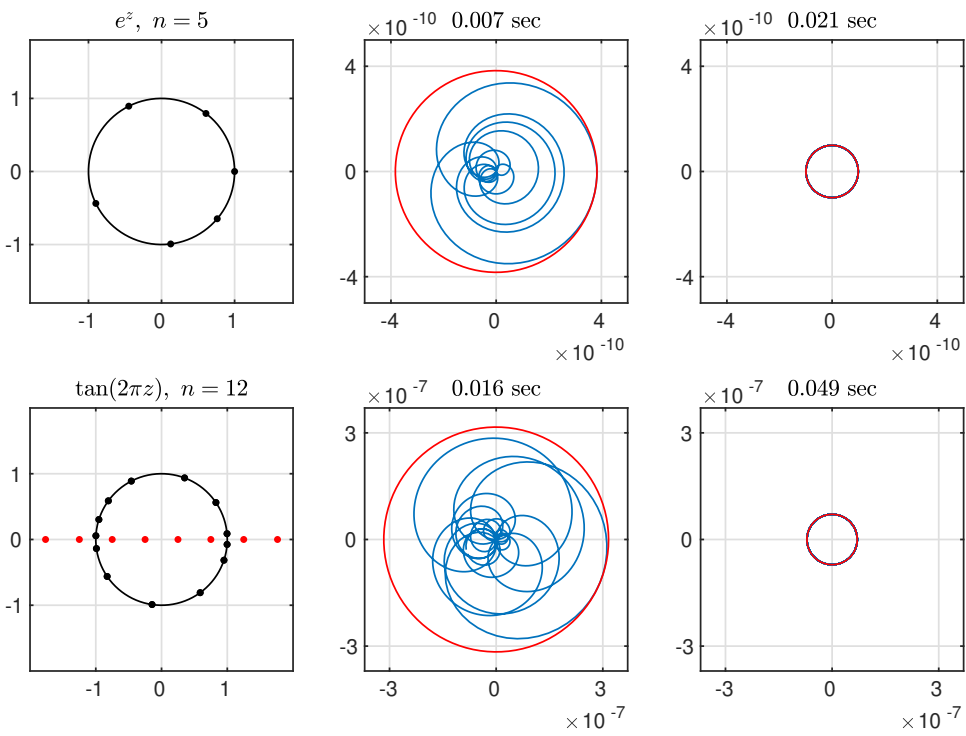

FIG. 4.1. Approximation on the unit circle of the analytic function $e^{z}$ and the meromorphic function $\tan (2 \pi z)$. The middle and right plots show error curves for AAA and AAA-Lawson approximation, respectively, with red circles marking the maximum errors. The minimax error curves are nearly circular (invisible here since they lie under the red circles), with winding numbers $2 n+1=11$ and $2 n+1-8=17$. Red and black dots mark poles and support points of the AAA-Lawson approximation. Color is available online only.

which establishes the lower bound $E^{*} \geq \sigma_{n+1}$, where $\left\{\sigma_{k}\right\}$ are the singular values of the infinite Hankel matrix of Taylor coefficients $a_{1}, a_{2}, \cdots=1,1 / 2 !, \ldots$ Here the relevant value is $\sigma_{6}=9.944144081 \mathrm{e}-11$.

Meromorphic function on the unit disk. The second example of Figure 4.1 is $\tan (2 \pi z)$ for $n=12$ in 1000 points of the unit circle. This function is meromorphic but not analytic in the unit disk. Again we get a nearly circular error curve, whose winding number is not 25 but 17 because of the four poles in the disk. Here AAALawson improves the error from 3.16e-7 to $7.08 \mathrm{e}-8$. The red dots in the left image mark poles of the AAA-Lawson approximation. The poles inside the circle match the poles $\pm 1 / 4$ and $\pm 3 / 4$ of $\tan (2 \pi z)$ to 13 digits of accuracy. We can explain this by noting that these poles can be determined by certain contour integrals of the boundary data [4, section 4], and since $r$ matches $\tan (2 \pi z)$ to many digits on the boundary, the contour integrals must match too. The poles of $r$ outside the circle are at \pm 1.250011 , $\pm 1.7638, \pm 2.6420$, and \pm 7.3844 . (In the first row of this figure, no red dots appear because the poles are off-scale. Their positions in the case of Padé approximations were investigated by Saff and Varga [67].)

Approximation on an ellipse and a square. Figure 4.2 shows approximations on two noncircular domains. In the first row, $\log (0.5-z)$ is approximated in 2000 points on an ellipse of half-height 1 and half-width 0.3 . Note how the poles of the approximation line up along the branch cut, a phenomenon analyzed for Padé approximations by Stahl [73]. It is also interesting to see that all the support points chosen by AAA lie on that side of the ellipse. The second row shows approximation of the Airy function $\operatorname{Ai}(2 z)$ [56] in 4000 points on the boundary of the unit square and 1000 points in a Chebyshev distribution on each side. The error curve, with winding number $2 n+1=21$, is nearly circular along most of its length, while retaining the four 

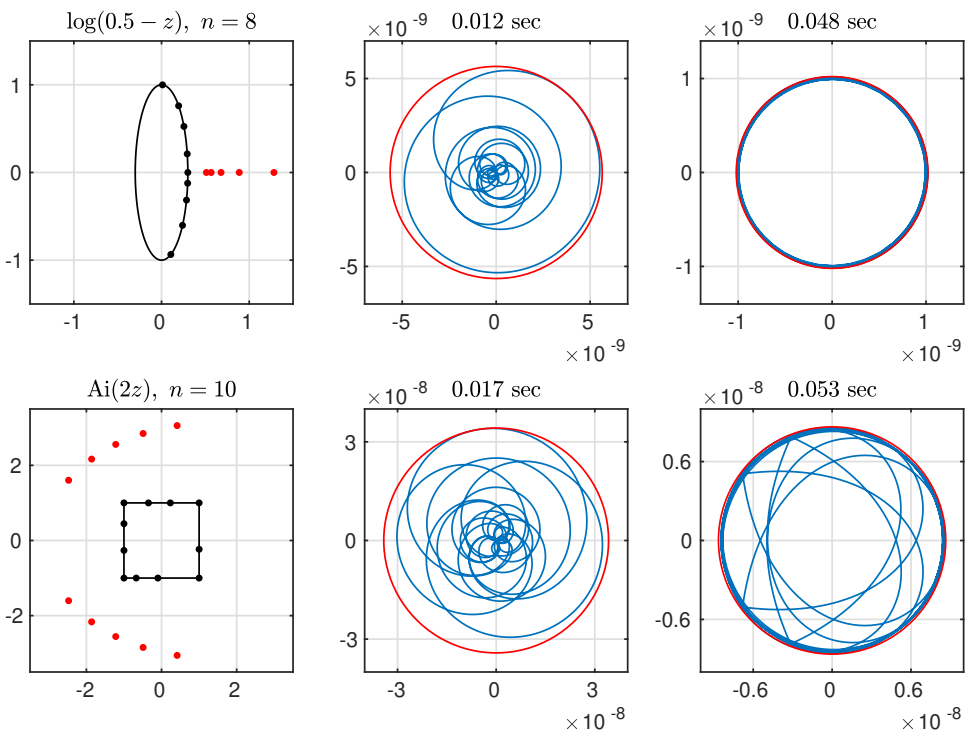

FIG. 4.2. Approximations on an ellipse and a square. The near-circularity effect appears again, though on the square, the four corners persist. Here and in most of figures to follow, the axis scales are different for the $A A A$ and $A A A$-Lawson plots.

corners associated with the square.

Approximation of branch point singularities. Figure 4.3 turns to problems with singularities on the boundary, where rational functions have their greatest power relative to polynomials, achieving root-exponential convergence as $n \rightarrow \infty$ by means of poles exponentially clustered near the singularities [29, 30, 31, 55]. In the first row, $\left(1+z^{4}\right)^{1 / 2}$ is approximated to degree $n=16$. The AAA-Lawson approximation improves the error from $1.38 \mathrm{e}-1$ to $6.49 \mathrm{e}-3$, with poles lying along branch cuts near each of the four singularities at radii 1.00046, 1.0085, 1.075, and 1.59. For successful computation of approximations with clustered poles like this, it is important that the sample grid be clustered too, and in this case the sample points on the unit circle were placed at angles $(\pi / 4) \cdot \tanh ($ inspace $(-12,12,1000)$ ) together with their rotations by angles $\pi / 2, \pi$, and $3 \pi / 2$. Note that there are four square roots in this function, hence four right angles in the error curve, but these appear as one because they lie on top of one another.

Conformal map of an L-shape. The second row of Figure 4.3 shows degree 20 approximation of an analytic function representing a conformal map of an L-shaped region onto the unit disk, which has a $z^{2 / 3}$ type of singularity at the reentrant corner. Each of the six sides has sample points with a distribution controlled by $\tanh ($ Iinspace $(-12,12,1000))$. In $[29]$, it was shown that AAA rational approximations of conformal maps of polygons can be 10-1000 times more efficient to evaluate than the standard method of Driscoll's Schwarz-Christoffel Toolbox [18]. From Figure 4.3 we see that even better approximations are available with AAA-Lawson, which improves the accuracy of the approximation in this case from $8.21 \mathrm{e}-4$ to $1.57 \mathrm{e}-4$.

Approximation on a discrete cloud of points. Figure 4.4 moves from essentially continuous domains to discrete ones consisting of random points in a rectangle. A rational function of degree $n$ could generically interpolate $2 n+1$ data values exactly. Thus the first nontrivial fit occurs with $2 n+2$ data values, and this is shown in the 

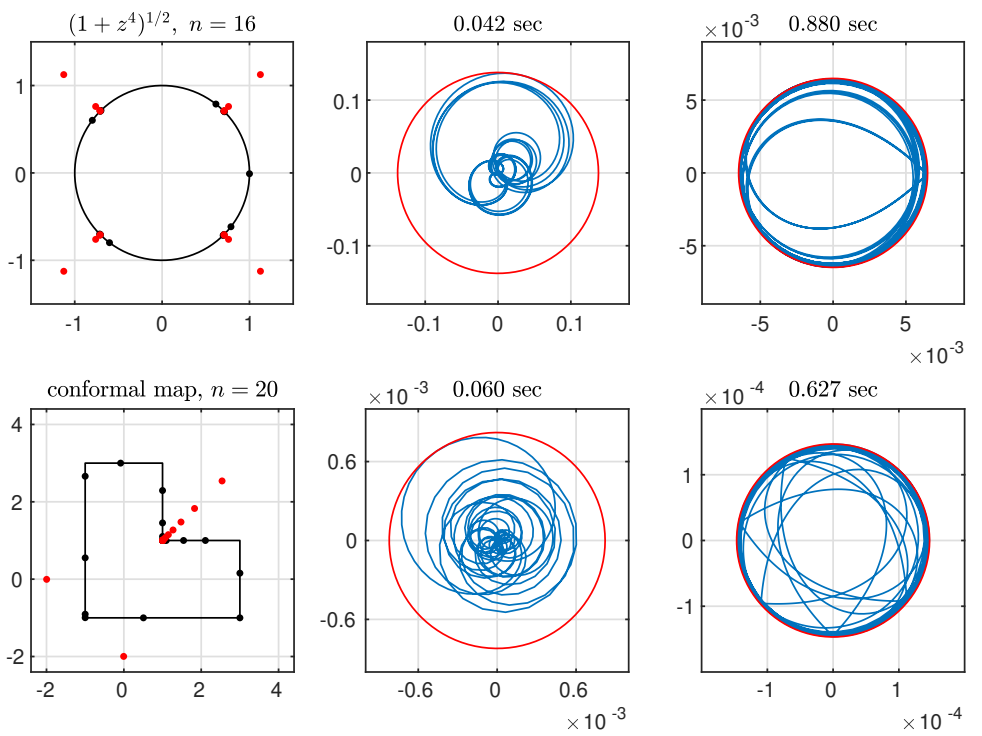

FIG. 4.3. Two approximation problems with singularities on the boundary. The second row, following [29], is the inverse of a Schwarz-Christoffel conformal map. Because of the prevalence of corner singularities, rational approximations can be a powerful tool in numerical conformal mapping and other areas of computational complex analysis.

first row of the figure, with $n=6$ and 14 sample points. As expected, the minimax error is attained at all 14 points. The second row increases the number of sample points to 100 , and now the maximum error, which is 10,000 times larger, is attained at 20 rather than 14 of them. (This is not evident with the calculation as run with the Chebfun default number of 20 Lawson steps, but emerges if a few hundred Lawson steps are taken to give convergence to more digits of accuracy.)

Approximation on complex arcs. Figure 4.5 shows two approximations on domains that are just arcs, a semicircle and an S-shape, both represented with 500 points in a Chebyshev distribution along each semicircular piece. Figure 4.6 shows, first, an approximation on the unit circle of a function with an essential singularity in the disk, and second, the clamped beam example from the NICONET model order reduction collection [12], which was also considered in [54]. (Another collection of more than 50 examples can be found at the Model-Order-Reduction-Wiki [52].) Here the approximation domain is the imaginary axis, which is discretized by 2000 points logarithmically spaced between $0.01 i$ and $100 i$ together with their complex conjugates. The function to be approximated is defined via the resolvent of a $348 \times 348$ matrix whose eigenvalues are in the left half-plane, making it analytic in the right half-plane. The rightmost pole pairs of the degree 12 rational approximation match those of the underlying matrix to accuracies of 5.5e-7, 9.2e-6, 1.1e-4, and 1.2e-4, counting in from the imaginary axis. Note that in this example, AAA-Lawson achieves reduction of the error in comparison with AAA by a factor of about 4, from 6.15 to 1.49. This is an improvement of the relative accuracy from $0.13 \%$ to $0.03 \%$, since the function being approximated takes values as large as 4550 .

Disconnected and multiply connected domains. Our final pair of complex examples, shown in Figure 4.7, involves domains of more complicated connectivity. The upper example approximates the function $\left(1-z^{-2}\right)^{1 / 2}$ on the boundary of the annu- 

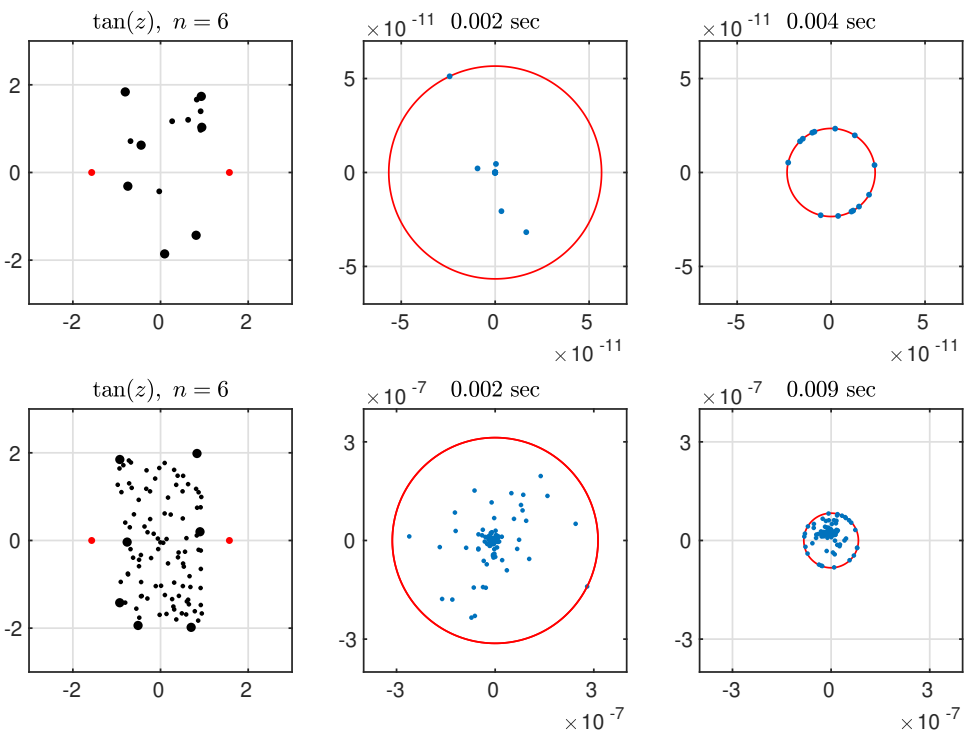

Fig. 4.4. Approximation of $\tan (z)$ at 14 and 100 random points in a rectangle in $\mathbf{C}$. In the first case, with just $2 n+2$ sample points, the minimax error is attained at every one.
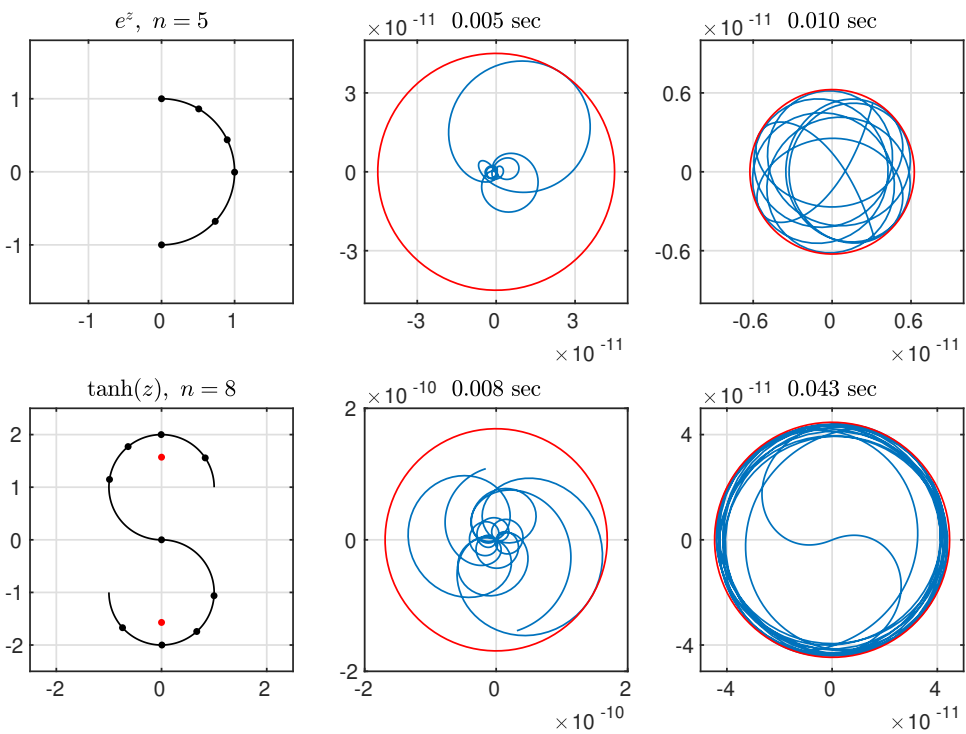

FIG. 4.5. Approximations on complex arcs.

lus $1 \leq|z| \leq 2$ (500 equispaced points on the outer circle together with 500 points each in a $\tanh (12 * 1$ inspace $(-1,1))$ distribution on the upper and lower halves of the inner circle). Note that as usual, the poles cluster near the singularities on the boundary, which in this case are at \pm 1 . The lower example approximates the function $z \operatorname{sign}(\operatorname{Re}(z))$ on the union of two circles of radius 1 about -1.5 and 1.5 (1000 equispaced points on each circle). This function is not globally analytic, and both AAA and AAA-Lawson tend to have difficulties with such problems. Indeed, if $z \operatorname{sign}(\operatorname{Re}(z))$ is replaced by $\operatorname{sign}(\operatorname{Re}(z))$, the iteration fails. Rational approximations on domains 

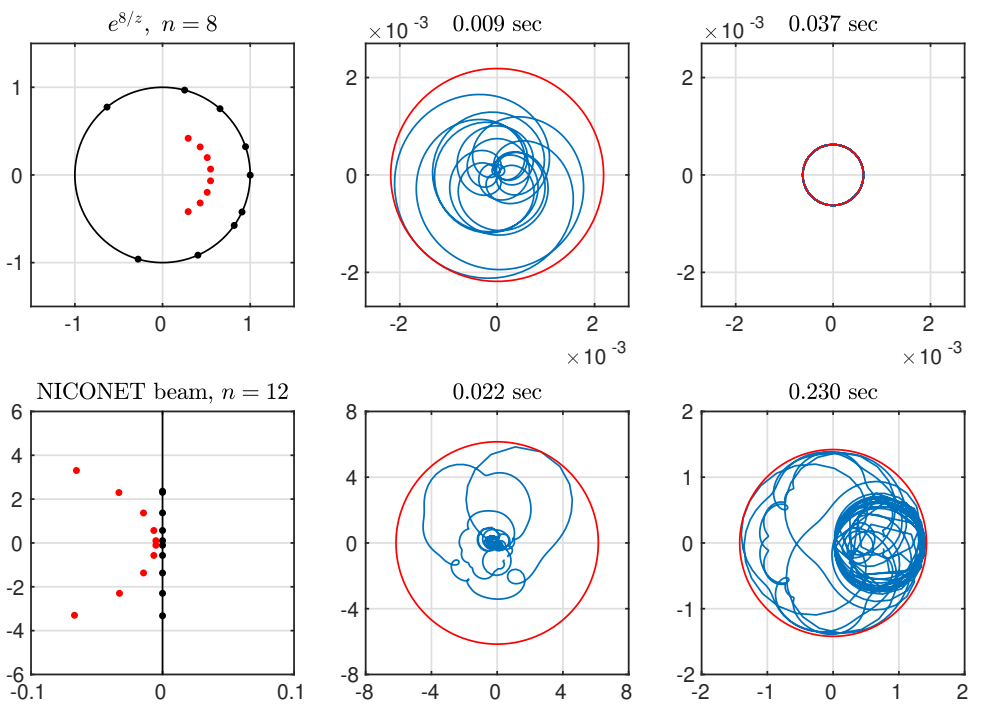

FIG. 4.6. The example of the first row has an essential singularity in the unit disk; all the poles fall inside the circle and the winding number is -17 . The second is the NICONET beam model order reduction example of [12], defined on the imaginary axis via the resolvent of a $348 \times 348$ matrix.
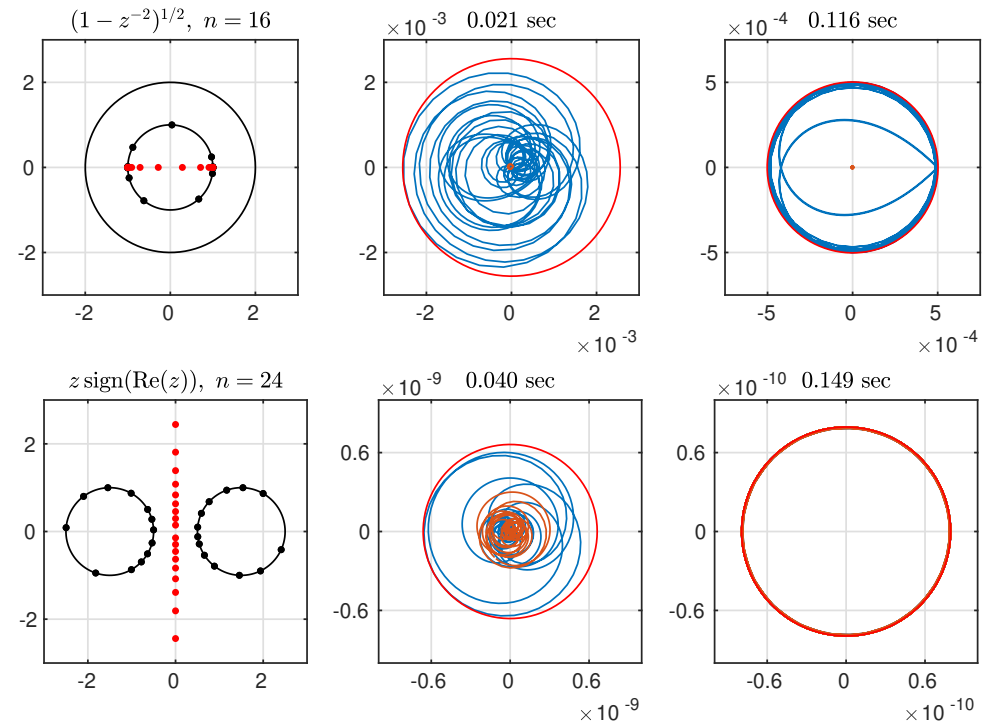

FIG. 4.7. Approximations on the boundaries of an annulus (doubly connected) and a union of disks (disconnected). In each case, the errors corresponding to the two disjoint boundary components are plotted in different colors. For the annulus, the small red mark near the origin reflects the fact that the best approximation has error 57.1 times smaller on the outer circle than the inner one. The two-disks example is related to problems of model order reduction and data compression [6, 46]. Color is available online only.

with two components arise in data compression and model order reduction [6, 46].

Reviewing the 14 AAA-Lawson error curves displayed in Figures 4.1-4.7 (or error dots in the case of Figure 4.4), we note that it seems vividly apparent from the nearmaximal values at most of the points that a near-minimax solution has been found; we 

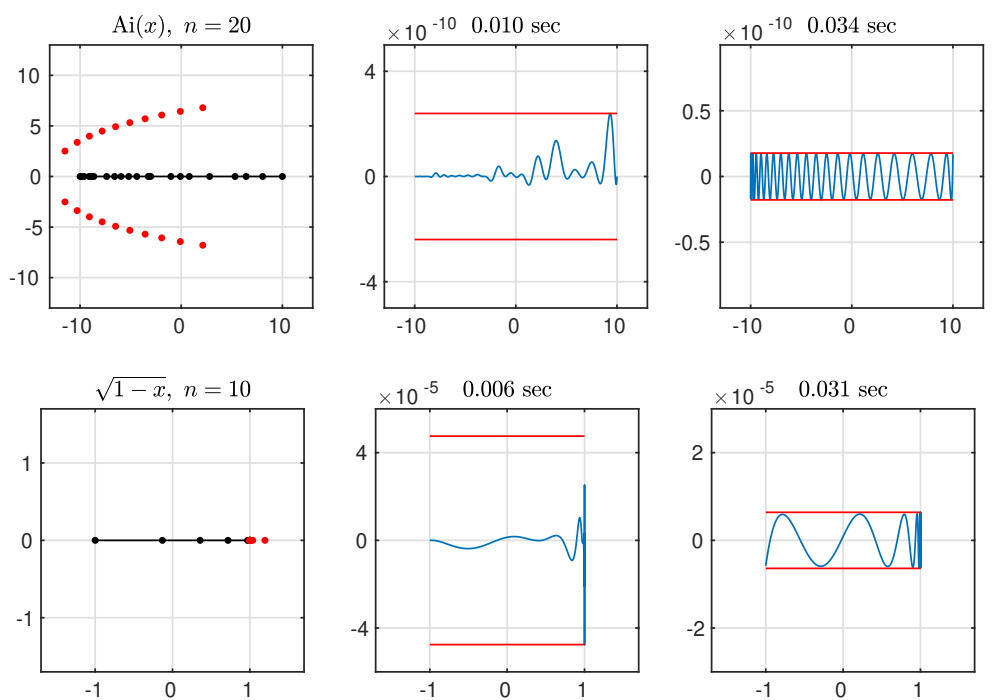

FIG. 5.1. Approximation on real intervals of an analytic function and a function with a singularity at one endpoint. The latter has exponentially clustered poles approaching within a distance $1.4 \mathrm{e}-8$ of $x=1$.

shall discuss the optimality question at the beginning of section 6 . Proving this would be a challenge, however, although the bound $E^{*} \geq \min _{z \in Z}|r(z)-f(z)|$ follows from arguments related to Rouché's theorem in cases where the error curve is a near-circle of sufficiently high winding number [35, 37, 41, 80].

5. Numerical examples, real. For real approximation on real intervals and unions of real intervals, AAA-Lawson, like AAA itself, is less reliable than in the complex case but retains its speed and flexibility. We shall present eight examples, grouping them again in pairs. Some comparisons are made with results from the Remez and CF algorithms (Chebfun minimax and cf, respectively), which differ in principle in working with a continuous interval rather than a discretization, though this difference is not very great in practice.

Analytic function on an interval. The first example of Figure 5.1 approximates the Airy function $\operatorname{Ai}(x)$ on $[-10,10]$, which is discretized by 1000 points in a Chebyshev distribution. Note how the poles lie along curves in the left half-plane, where the function is larger. (The study of such curves in approximation theory goes back to an investigation of roots of Taylor polynomials of $e^{z}$ by Szegö [74].) In this case of an analytic function on a single interval, Chebfun's minimax gets the answer in 1.7 secs. and its CF command cf does it in just 0.05 secs. [85]. The second example of the figure considers $(1-x)^{1 / 2}$, which has a singularity at the right endpoint, discretized on the grid $\tanh ($ Iinspace $(-12,12,1000))$. The poles of this approximation cluster near $x=1$ at distances $15.3,2.1,0.19,3.7 \mathrm{e}-2,6.4 \mathrm{e}-3,9.5 \mathrm{e}-4,1.1 \mathrm{e}-4,1.0 \mathrm{e}-5,5.9 \mathrm{e}-7$, and 1.4e-8. Chebfun minimax is unsuccessful for this problem with $n=10$, though it can handle degrees up to $n=7$.

Interior singularities and near-singularities. Figure 5.2 turns to functions with a singularity or near-singularity in the interior of the interval. We pick two examples where AAA and AAA-Lawson are successful, though failures are common with problems of this kind. The first example is $|x|$, the problem made famous by Donald Newman, which is discretized by transplants of $\tanh (\operatorname{linspace}(-12,12))$ to both 

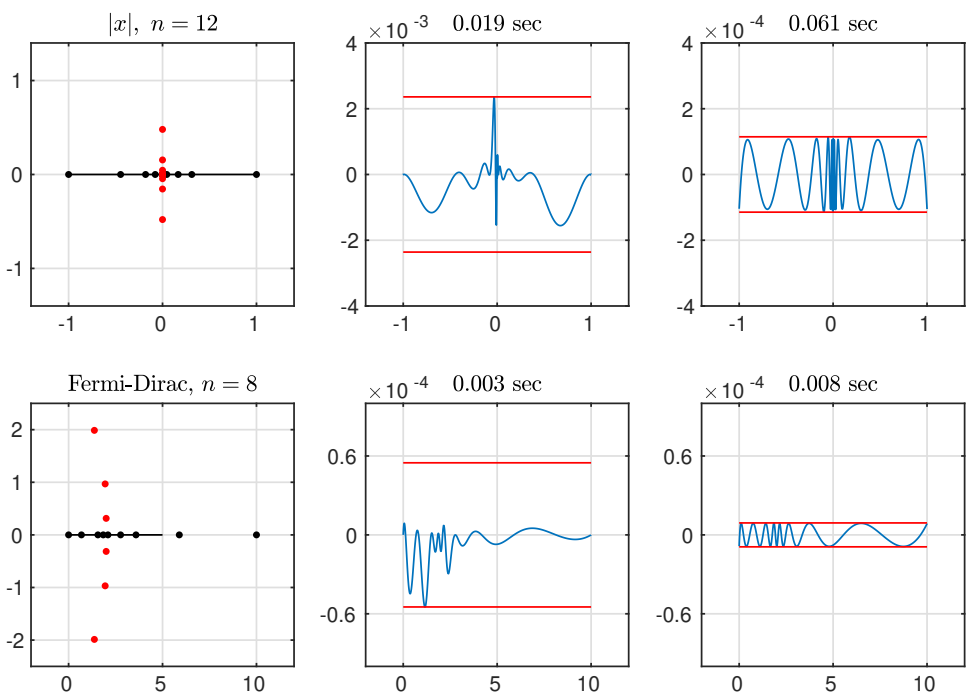

FIG. 5.2. Approximation of functions with singularities (above) and near-singularities (below) in the interval of approximation. The Fermi-Dirac problem comes from electronic structure calculation [53].

$[-1,0]$ and $[0,1]$; see [55] and [81, Chapter 25]. The AAA-Lawson error of 1.23e-4 is a bit higher than the result 1.07e- 4 computed by Chebfun minimax in 1.2 secs. As in Figures 4.2 and 4.3 , we see the 12 poles lining up along a branch cut; their locations are approximately $\pm 0.00138 i, \pm 0.0102 i, \pm 0.0448 i, \pm 0.155 i, \pm 0.4780 i$, and $\pm 1.98 i$. The second is the Fermi-Dirac function $1 /(1+\exp (10(x-2)))$ on the interval $[0,10]$, as discussed, for example, in $[48,53]$, for which AAA-Lawson gets an error of 9.09e-6. Chebfun minimax gets the better value $8.77 \mathrm{e}-6$ in 0.2 secs., and cf does the same in 0.05 secs. (A more robust computational strategy for Fermi-Dirac functions is to first transplant $[0, \infty)$ to $[-1,1]$ by a Möbius transformation $[53,82]$.)

Approximation on a union of intervals. Figure 5.3 considers a pair of problems on a union of two intervals, $[-3,-1] \cup[1,3]$, each discretized by 500 points in a Chebyshev distribution. The first function, $\sin (6 x)$, is globally analytic, but the second, $|x| \sin (x)$, is not. Note how the poles line up along the imaginary axis, delineating once more an implicit branch cut.

Approximation on infinite intervals. The final pair of examples, shown in Figure 5.4, are posed on infinite intervals. The first is the Cody-Meinardus-Varga problem of approximation of $e^{x}$ on $(-\infty, 0][15,20,54,84,81]$. As described in section 4 of [84], one can compute approximations for this problem by transplantation of $(-\infty, 0]$ to $[-1,1]$ followed by $\mathrm{CF}$ or minimax approximation, but here we approximate directly on the original untransplanted interval, which is discretized by 2000 points logarithmically spaced from $-10^{6}$ to $-10^{-6}$. The success of such a computation highlights the extraordinary flexibility and stability of barycentric representations based on support points selected by AAA. The second example of Figure 5.4 shows approximation of $\exp \left(-x^{2}\right)$ on $(-\infty, \infty)$, discretized by 100 equispaced points in $[-1,1]$ concatenated with 500 logarithmically spaced points in $\left[1,10^{6}\right]$ and their negatives in $\left[-10^{6},-1\right]$. AAA-Lawson improves the error from $6.92 \mathrm{e}-6$ to $1.04 \mathrm{e}-6$.

In general, we believe that the safest way to compute a real minimax approximation on a real interval is usually by the Remez algorithm as implemented in Chebfun 

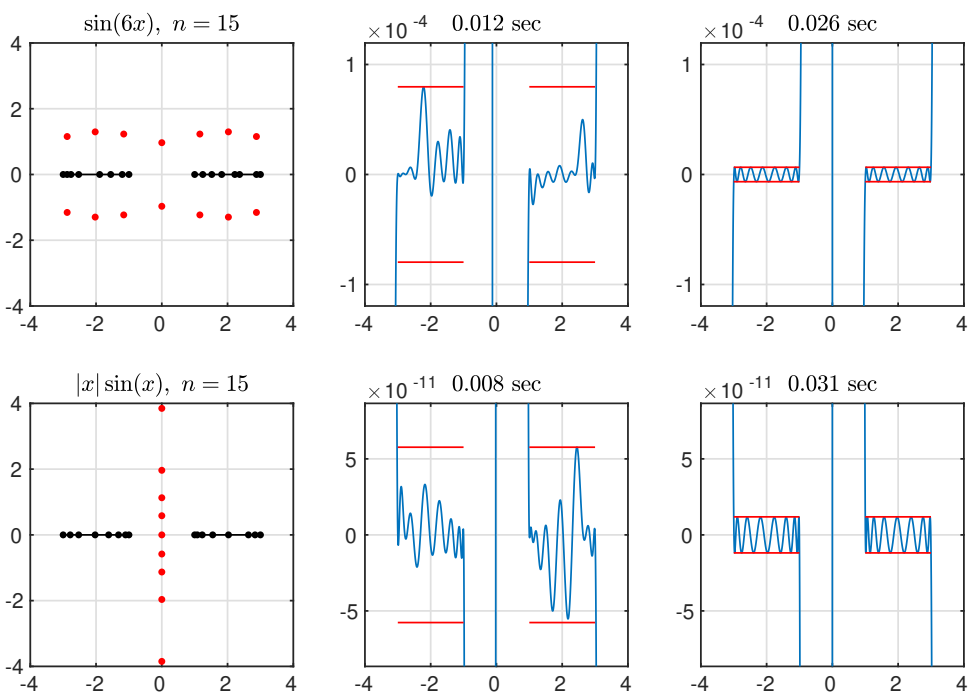

FIG. 5.3. Approximation of two functions each on a pair of disjoint intervals. The first example, $\sin (6 x)$, is globally analytic, whereas the second, $|x| \sin (x)$, is analytic on each interval but not globally. The very different configurations of poles reflect this distinction.
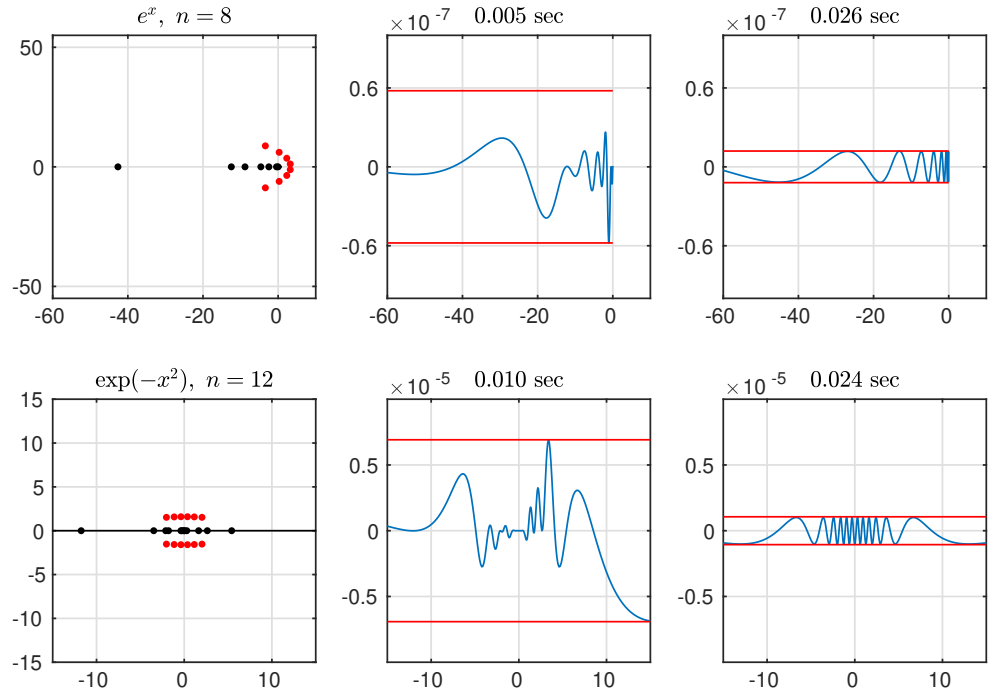

FIG. 5.4. Approximations on $(-\infty, 0]$ and $(-\infty, \infty)$. The first example is the starting point of the use of rational approximations for numerical solution of partial differential equations [15, 20, 84].

minimax, or if the function is smooth, by $\mathrm{CF}$ approximation as implemented in cf, in both cases perhaps after softening up the problem by a Möbius transformation. The AAA-Lawson approach is most important in cases where these simple tools are inapplicable, such as unbounded or disjoint intervals as in Figure 5.3 or 5.4.

6. Convergence properties. Our experience with applying the AAA-Lawson method to hundreds of examples can be summarized as follows: for analytic functions on well-resolved complex domains, it almost always converges to the minimax solution, and for nonanalytic functions or real domains, it often does so. The first question 
that needs to be answered about these claims is, how do we know that convergence has successfully occurred? There is no mathematical guarantee that applies in all cases, which would indeed be a challenge for any algorithm in the absence of effective criteria for global optimality, and we do not attempt to check local optimality criteria numerically. Nevertheless, there is evidence of various kinds. For real approximations on intervals, there is the equiosillation characterization, which applies to the examples of section 5. For smooth approximations on complex domains, an error curve is often found that is virtually circular and of high enough winding number, in which case nearoptimality is guaranteed by arguments related to Rouché's theorem, as mentioned at the end of section 4. This applies to the examples of Figure 4.1, the first examples of Figures 4.2 and 4.6, and the second example of Figure 4.7. In other cases, including most of the rest of the examples of section 4, the error curve is nearly circular over most of its range, suggesting near-optimality without providing a proof. Finally, there is the empirical evidence, as mentioned in the introduction, that the AAALawson algorithm successfully reproduces all 29 examples computed by Ellacott and Williams [22] by a different method.

To give some details about the caveat that the problem should involve analytic functions on well-resolved complex domains, we now summarize five contexts in which we have found that AAA-Lawson is most likely to fail. After this summary, we will turn to a particularly interesting aspect of potential nonconvergence.

1. Discretization too coarse. Most applications involve discretization of a continuum, and trouble often arises if the discretization is too coarse, especially near singular points where poles need to accumulate. Perhaps this is unsurprising since even existence of best approximations fails in general on discrete domains, as mentioned in section 2. As indicated in the discussion of examples in the last two sections, we routinely use Chebyshev-type sample point clustering near nonsingular corners or endpoints of domains and more extreme $\tanh ($ linspace $(-12,12, \mathrm{npts})$ )-type clustering near singular points.

2. Too close to machine precision. By default, AAA delivers an approximation with accuracy close to machine precision, and attempted Lawson iterations from such a point tend to take on a random character, leading to failure. Instead, in standard double precision arithmetic, it is best to use AAA-Lawson for approximations with errors down to $10^{-12}$ or $10^{-13}$ but not much smaller. The Chebfun aaa code reflects this by running without Lawson if no degree is specified, e.g., aaa $(F, Z)$, and with Lawson if a degree is specified, e.g., aaa (F, Z, 'degree', 10$)$. These defaults can be overridden by specifying aaa (..., 'lawson', nsteps) in which case exactly nsteps Lawson steps are taken, and none at all if nsteps $=0$. When we want to compute the minimax approximation to greater accuracy than is delivered by the default parameters, we specify a large value of nsteps.

3. Degeneracy related to symmetry. Failure often occurs if one attempts a calculation that does not respect the symmetry of the problem, where the mathematically correct best approximation is degenerate. For example, an attempt to compute a degree 3 best approximation to $\exp \left(z^{2}\right)$ on the unit disk will fail, because the result should be of degree 2 . If the degree specification is changed to 2 , the calculation succeeds.

4. Lack of analyticity. The examples of section 4 illustrated that AAA-Lawson has little trouble with functions meromorphic in a disk or an annulus. Failures often occur in the approximation of more deeply nonanalytic functions, however. For example, the problem with 100 random points of Figure 4.4 fails if $f(z)$ is changed from $\tan (z)$ to $|z|$. 

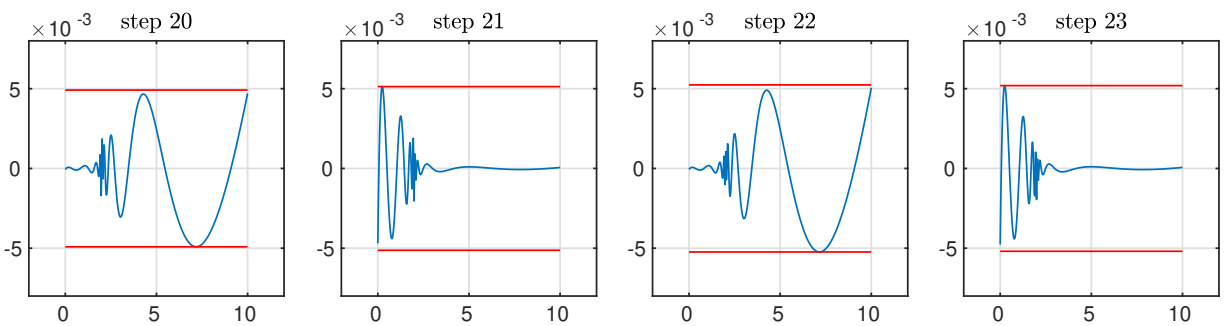

FIG. 6.1. Convergence failure sometimes takes the form of period-2 oscillations in the Lawson iteration. The effect is illustrated here for the Fermi-Dirac problem of Figure 5.2, but with the transition steepness parameter increased from 10 to 50.

5. Real domains. Failures are also common in approximation of real functions on real domains. As discussed in [54], such problems are difficult for AAA itself.

We now mention a particular failure mode we have observed that is perhaps of special mathematical interest: period-2 oscillations. Sometimes, even with an analytic function on a seemingly well-resolved grid, the Lawson iteration enters into a cycle in which one pattern of weights and errors appears at odd steps and another at even ones. For example, as shown in Figure 6.1, this happens with the Fermi-Dirac example of Figure 5.2 if $1 /(1+\exp (10(x-2)))$ is changed to $1 /(1+\exp (50(x-2)))$. We think this behavior results from fundamental mathematical properties associated with the discrete weighted $\ell_{2}$ norm of (3.6) and (3.7), and that fixing the problem in a principled way will require further theoretical and algorithmic developments. The issues in play may be related to the nonconvergence phenomena for nonlinear Lawson iterations investigated by Guoyong Shi in [69]. As a practical matter, one can usually fix the problem by underrelaxation in the update formula (3.7), or by a better distribution of sample points - here by putting a larger fraction of points in the transition region - but of course it would be much better to find a formulation that enabled convergence without such human intervention. We regard this as an important matter for further research.

As an engineering precaution against failure, the Chebfun code currently reverts to the AAA solution if AAA-Lawson fails to make an improvement.

Another convergence puzzle to be investigated concerns the successful linear convergence of the AAA-Lawson iteration in problems with nearly circular error curves, as in Figure 4.1. Here the nearly constant error has the effect that the Lawson weight distribution virtually stops changing from step to step [78], and in particular, effectively never gets close to the sum of delta functions form that an asymptotic analysis is likely to look for $[14,44]$. Nevertheless, the approximations in such cases often converge quickly, and we have observed that they converge much faster still if (3.7) is modified to depend on $\left|e_{j}\right|^{2}$ instead of $\left|e_{j}\right|$ (cf. [21, sect. 5]), although in other cases this modification results in failure. Perhaps a deeper understanding of the convergence properties of the AAA-Lawson iteration may shed light on these effects.

7. Comparison with Ellacott-Williams and Istace-Thiran algorithms. As has been mentioned, Ellacott-Williams (EW) and Istace-Thiran (IT) algorithms for complex rational minimax approximation have been published previously by Ellacott and Williams [22] and Istace and Thiran [40], building on earlier work by Osborne and Watson [60]. In this section, we comment on the relationship of these to the AAA-Lawson algorithm.

The EW and IT algorithms start from the partial characterizations of best ap- 
proximations mentioned in section 2. The local Kolmogorov criterion asserts that if $r=p / q$ is a local best approximation, then

$$
\left\|f-\frac{p}{q}\right\| \leq\left\|f-\frac{p+\delta p}{q+\delta q}\right\|
$$

for all sufficiently small polynomial perturbations $\delta p$ and $\delta q$. Linearizing gives

$$
\frac{p+\delta p}{q+\delta q} \approx \frac{p}{q}+\frac{q \delta p-p \delta q}{q^{2}}
$$

and this suggests the following iteration. Determine an initial guess $r_{0}=r_{0} / q_{0}$, and then define

$$
r_{k+1}=\frac{p_{k}}{q_{k}}+\frac{q_{k} \delta p_{k}-p_{k} \delta q_{k}}{q_{k}^{2}}, \quad k=0,1, \ldots,
$$

by the condition that $r_{k+1}$ minimizes $\left\|f-r_{k+1}\right\|$ over polynomials $\delta p_{k}$ and $\delta q_{k}$. Since $q_{k}$ is known, this is a problem of linear minimax approximation. If $\delta p_{k}$ and $\delta q_{k}$ can be calculated, they define what will usually be a descent direction for the nonlinear problem of minimizing $\|f-r\|$, and the actual choice of $r_{k+1}$ may then be improved by a line search in this direction.

Both the EW and the IT algorithms aim to execute an iteration of this kind until convergence to a suitable tolerance; of course we are omitting numerous details. One of the differences between the algorithms consists in how the minimization subproblem is solved. Ellacott and Williams do this via a linear Lawson iteration, making their algorithm potentially slow because it contains an inner iteration embedded in the outer iteration. Istace and Thiran propose the use of Tang's Remez-type Chebyshev approximation algorithm instead [75]. This is potentially quicker, with quadratic instead of linear convergence. A disadvantage is that a derivative of the error function is required, which may not be available in applications where $f$ is unknown and can only be sampled.

This outline highlights four ways in which AAA-Lawson differs from EW and IT:

1. The outer iteration converges linearly, not quadratically.

2. There is no inner iteration, just a linear least-squares problem.

3. The initial guess comes from the AAA algorithm.

4. The rational function is represented in barycentric form.

These are substantial differences, and in the absence of software for EW and IT, it is unclear how most appropriately to compare AAA-Lawson against them. As just a partial step, we have made comparisons with our own implementation of the EW method. First we used the initial guess $r_{0}$ proposed by Ellacott and Williams, based on a linear minimax problem, but it proved much more reliable, following (3), to get $r_{0}$ from the AAA algorithm. With this improvement, we have been able to solve about half the problems of sections 4 and 5 by the EW algorithm. (We can also solve all 29 of the EW test problems, but these are relatively easy examples involving low degrees $\leq 5$ and simple functions on the disk, the half-disk, or the quarter-disk.) In some of the unsuccessful cases, there appears to be convergence to a local minimum that is not the global minimum.

We believe that one reason for convergence difficulties of EW is its use of a $p / q$ representation, with $p$ and $q$ each represented in the monomial basis. Some improvement could probably be achieved by the use of other bases when the domain is not a disk, calculated perhaps on-the-fly by the Vandermonde-with-Arnoldi method [11]. 
However, we suspect a greater improvement might be achieved if the algorithm could be converted to a barycentric form so as to eliminate the difference (4) in the list above. Explanations of the great difference in stability between $p / q$ and barycentric representations can be found in [23, sect. 2] and [54, sect. 11]. The development of a barycentric-EW or barycentric-IT algorithm would be a very interesting subject for investigation.

8. Discussion. The AAA-Lawson algorithm makes it easy for the first time to compute real and complex minimax rational approximations on all kinds of domains. In Chebfun, for example, the commands

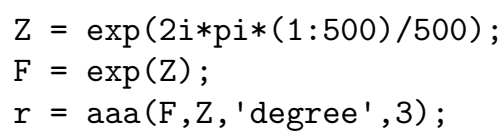

produce a function handle $\mathrm{r}$ for the best degree 3 rational approximation of $f$ on the unit circle in 0.05 secs. on the computer specified in section 4 . The calculation norm $(F-r(Z)$, inf $)$ then gives $9.9318 \mathrm{e}-6$, matching the result published in [22] many years ago.

What makes the algorithm so effective is that it combines the exceptional stability of barycentric rational representations, as exploited by the AAA algorithm [54], with the long-established technique of IRLS iteration to improve the error to minimaxthough in a novel nonlinear barycentric context. It is interesting that, unlike its predecessors by Ellacott and Williams [22] and Istace and Thiran [40], AAA-Lawson is not based on an attempt to satisfy optimality conditions.

As discussed in the last two sections, AAA-Lawson has little theoretical foundation at present, and it also suffers from just linear asymptotic convergence, sometimes at a low rate. These drawbacks of IRLS iteration have been recognized for many years, as can be seen in this quote from p. 50 of Osborne's book of 1985 [59]:

The evidence presented here does not provide a recommendation for the technique [IRLS]. It is shown that the convergence rate is only first order in general and that even this cannot be guaranteed.

And yet, thanks to its simplicity and lack of dependence on a characterization of optimal solutions, IRLS has enabled us to develop an algorithm that appears fast and robust. It is a challenge for the future to develop further improvements to the algorithm that might ensure its convergence in all circumstances and to support these with theoretical guarantees.

This article has considered only standard minimax approximations, without weight functions. Nonconstant weights are easily introduced by modifying (3.7). Another restriction is that we have treated only rational approximations of type $(n, n)$, not type $(m, n)$ with $m \neq n$. The more general problem is certainly interesting, and AAA itself can be generalized to $m \neq n$ as described in [54]. However, though the "Walsh table" of approximations of a function of all rational types is fascinating, the overwhelming majority of applications are concerned with types $(n, n)$ or $(n-1, n)$.

Acknowledgments. We are grateful to Silviu Filip and Abi Gopal for assistance with both software and mathematics. 


\section{REFERENCES}

[1] B. Alpert, L. Greengard, and T. Hagstrom, Rapid evaluation of nonreflecting boundary kernels for time-domain wave propagation, SIAM J. Numer. Anal., 37 (2000), pp. 11381164, https://doi.org/10.1137/S0036142998336916.

[2] A. C. Antoulas, Approximation of Large-Scale Dynamical Systems, SIAM, Philadelphia, 2005, https://doi.org/10.1137/1.9780898718713.

[3] A. C. Antoulas, S. Lefteriu, and A. C. Ionita, A tutorial introduction to the Loewner framework for model reduction, in Model Reduction and Approximation: Theory and Algorithms, Comput. Sci. Eng 15, SIAM, Philadelphia, 2017, pp. 335-376, https://doi. org/10.1137/1.9781611974829.ch8.

[4] A. P. Austin, P. Kravanja, and L. N. Trefethen, Numerical algorithms based on analytic function values at roots of unity, SIAM J. Numer. Anal., 52 (2014), pp. 1795-1821, https: //doi.org/10.1137/130931035.

[5] I. Barrodale, L. M. Delves, And J. C. Mason, Linear Chebyshev approximation of complexvalued functions, Math. Comp., 32 (1978), pp. 853-863.

[6] B. Beckermann And A. Townsend, Bounds on the singular values of matrices with displacement structure, SIAM Rev., 61 (2019), pp. 319-344, https://doi.org/10.1137/19M1244433.

[7] P. Benner, A. Cohen, M. Ohlberger, and K. Willcox, eds., Model Reduction and Approximation: Theory and Algorithms, SIAM, Philadelphia, 2017, https://doi.org/10.1137/ 1.9781611974829.

[8] M. BerlJAFA AND S. GÜtTel, The RKFIT algorithm for nonlinear rational approximation, SIAM J. Sci. Comput., 39 (2017), pp. A2049-A2071, https://doi.org/10.1137/15M1025426.

[9] J.-P. Berrut and L. N. Trefethen, Barycentric Lagrange interpolation, SIAM Rev., 46 (2004), pp. 501-517, https://doi.org/10.1137/S0036144502417715.

[10] D. Braess, Nonlinear Approximation Theory, Springer, Berlin, 1986.

[11] P. D. Brubeck, Y. Nakatsukasa, and L. N. Trefethen, Vandermonde with Arnoldi, SiAM Rev., to appear.

[12] Y. Chahlaoui and P. Van Dooren, A Collection of Benchmark Examples for Model Reduction of Linear Time Invariant Dynamical Systems, EPrint 2008.22, Manchester Institute for Mathematical Sciences, University of Manchester, Manchester, UK, 2002.

[13] X. Chen and T. W. Parks, Design of IIR filters in the complex domain, IEEE Trans. Acoust. Speech Signal Process., 38 (1990), pp. 910-920.

[14] A. K. Cline, Rate of convergence of Lawson's algorithm, Math. Comp., 26 (1972), pp. 167-176.

[15] W. J. Cody, G. Meinardus, and R. S. Varga, Chebyshev rational approximation to $e^{-x}$ in $[0,+\infty)$ and applications to heat-conduction problems, J. Approximation Theory, 2 (1969), pp. 50-65.

[16] P. Cooper, Rational Approximation of Discrete Data with Asymptotic Behaviour, Ph.D. thesis, University of Huddersfield, Huddersfield, England, 2007.

[17] I. Daubechies, R. DeVore, M. Fornasier, And C. S. Güntürk, Iteratively reweighted least squares minimization for sparse recovery, Comm. Pure Appl. Math., 63 (2010), pp. 1-38.

[18] T. A. Driscoll, A MATLAB toolbox for Schwarz-Christoffel mapping, ACM Trans. Math. Software, 22 (1996), pp. 168-186.

[19] T. A. Driscoll, N. Hale, and L. N. Trefethen, eds., Chebfun User's Guide, Pafnuty Publications, Oxford, UK, 2014; also available online from http://www.chebfun.org.

[20] V. Druskin, L. Knizhnerman, and M. Zaslavsky, Solution of large scale evolutionary problems using rational Krylov subspaces with optimized shifts, SIAM J. Sci. Comput., 31 (2009), pp. 3760-3780, https://doi.org/10.1137/080742403.

[21] S. Ellacott and J. Williams, Linear Chebyshev approximation in the complex plane using Lawson's algorithm, Math. Comp., 30 (1976), pp. 35-44.

[22] S. Ellacott and J. Williams, Rational Chebyshev approximation in the complex plane, SIAM J. Numer. Anal., 13 (1976), pp. 310-323, https://doi.org/10.1137/0713028.

[23] S.-I. Filip, Y. Nakatsukasa, L. N. Trefethen, and B. Beckermann, Rational minimax approximation via adaptive barycentric representations, SIAM J. Sci. Comput., 40 (2018), pp. A2427-A2455, https://doi.org/10.1137/17M1132409.

[24] B. Fischer AND J. ModersitZKI, An algorithm for complex linear approximation based on semi-infinite programming, Numer. Algorithms, 5 (1993), pp. 287-297.

[25] M. S. Floater And K. Hormann, Barycentric rational interpolation with no poles and high rates of approximation, Numer. Math., 107 (2007), pp. 315-331.

[26] D. GaIER, Lectures on Complex Approximation, Birkhäuser, Boston, MA, 1987.

[27] T. W. Gamelin, Uniform Algebras, Prentice-Hall, Englewood Cliffs, NJ, 1969.

[28] K. Glashoff and K. RolefF, A new method for Chebyshev approximation of complex-valued

Copyright (C) by SIAM. Unauthorized reproduction of this article is prohibited. 
functions, Math. Comp., 36 (1981), pp. 233-239.

[29] A. Gopal and L. N. Trefethen, Representation of conformal maps by rational functions, Numer. Math., 142 (2019), pp. 359-382.

[30] A. Gopal and L. N. Trefethen, New Laplace and Helmholtz solvers, Proc. Natl. Acad. Sci. USA, 116 (2019), pp. 10223-10225.

[31] A. Gopal And L. N. TREFEthen, Solving Laplace problems with corner singularities via rational functions, SIAM J. Numer. Anal., 57 (2019), pp. 2074-2094, https://doi.org/10.1137/ 19M125947X.

[32] S. Gugercin, A. C. Antoulas, and C. Beattie, $\mathcal{H}_{2}$ model reduction for large-scale linear dynamical systems, SIAM J. Matrix Anal. Appl., 30 (2008), pp. 609-638, https://doi.org/ $10.1137 / 060666123$.

[33] B. Gustavsen and A. Semlyen, Rational approximation of frequency domain responses by vector fitting, IEEE Trans. Power Deliv., 14 (1999), pp. 1052-1061.

[34] M. H. Gutknecht, Non-strong uniqueness in real and complex Chebyshev approximation, J. Approx. Theory, 23 (1978), pp. 204-213.

[35] M. H. Gutknecht, Algebraically Solvable Approximation Problems, manuscript, http://people. maths.ox.ac.uk/trefethen/gutknecht83.pdf, 1983.

[36] M. H. Gutknecht And L. N. Trefethen, Nonuniqueness of best rational Chebyshev approximations on the unit disk, J. Approx. Theory, 39 (1983), pp. 275-288.

[37] E. Hayashi, L. N. Trefethen, and M. H. Gutknecht, The CF table, Constr. Approx., 6 (1990), pp. 195-223.

[38] P. HenricI, Applied and Computational Complex Analysis, Vol. 1, Wiley, New York, London, Sydney, 1974.

[39] A. C. Ioniță, Lagrange Rational Interpolation and its Applications to Approximation of LargeScale Dynamical Systems, Ph.D. thesis, Rice University, Houston, TX, 2013.

[40] M.-P. Istace and J.-P. Thiran, On computing best Chebyshev complex rational approximants, Numer. Algorithms, 5 (1993), pp. 299-308.

[41] V. KLOTZ, Gewisse rationale Tschebyscheff-Approximationen in der komplexen Ebene, J. Approximation Theory, 19 (1977), pp. 51-60.

[42] A. N. Kolmogorov, A remark on the polynomials of P. L. Chebyshev deviating the least from a given function, Uspehi Matem. Nauk (N.S.), 3 (1948), pp. 216-221 (in Russian).

[43] W. Krabs And G. Opfer, Eine Method zur Lösung des komplexen Approximationsproblem mit einer Anwendung auf konforme Abbildungen, Z. Angew. Math. Mech., 55 (1975), pp. T208$\mathrm{T} 211$.

[44] C. L. Lawson, Contributions to the Theory of Linear Least Maximum Approximations, Ph.D. thesis, UCLA, Los Angeles, CA, 1961.

[45] C. M. LEE AND F. D. K. RoBeRTs, A comparison of algorithms for rational $\ell_{\infty}$ approximation, Math. Comp., 27 (1973), pp. 111-121.

[46] J.-R. Li AND J. White, Low-rank solution of Lyapunov equations, SIAM Rev., 46 (2004), pp. 693-713, https://doi.org/10.1137/S0036144504443389.

[47] P. Lietaert, K. Meerbergen, J. Pérez, and B. Vandereycken, Automatic Rational Approximation and Linearization of Nonlinear Eigenvalue Problems, preprint, https://arxiv. org/abs/1801.08622, 2018.

[48] L. Lin, M. Chen, C. YAng, AND L. He, Accelerating atomic orbital-based electronic structure calculation via pole expansion and selected inversion, J. Phys. Condens. Matt., 25 (2019), 295501.

[49] K. N. Lungu, Best approximations by rational functions, Math. Notes, 10 (1971), pp. 431-433.

[50] H. J. Maehly, Methods for fitting rational approximations, parts II and III, J. Assoc. Comput. Mach., 10 (1963), pp. 257-277.

[51] G. Meinardus and D. Schwedt, Nicht-lineare Approximationen, Arch. Ration. Mech. Anal., 17 (1964), pp. 297-326.

[52] Model-Order-Reduction-Wiki, https://morwiki.mpi-magdeburg.mpg.de/morwiki.

[53] J. E. Moussa, Minimax rational approximation of the Fermi-Dirac distribution, J. Chem. Phys., 145 (2016), 164108.

[54] Y. Nakatsukasa, O. Sète, and L. N. Trefethen, The AAA algorithm for rational approximation, SIAM J. Sci. Comput., 40 (2018), pp. A1494-A1522, https://doi.org/10.1137/ 16M1106122.

[55] D. J. Newman, Rational approximation to $|x|$, Michigan Math. J., 11 (1964), pp. 11-14.

[56] F. W. J. Olver, D. W. Lozier, R. F. Boisvert, and C. W. Clark, NIST Handbook of Mathematical Functions, Cambridge University Press, New York, 2010.

[57] G. Opfer, An algorithm for the construction of best approximations based on Kolmogorov's criterion, J. Approx. Theory, 23 (1978), pp. 299-317.

Copyright $@$ by SIAM. Unauthorized reproduction of this article is prohibited. 
[58] A. V. Oppenheim and R. W. Schafer, Discrete-Time Signal Processing, Pearson, Noida, India, 2014

[59] M. R. Osborne, Finite Algorithms in Optimization and Data Analysis, Wiley, Chichester, 1985.

[60] M. R. Osborne And G. A. Watson, An algorithm for minimax approximation in the nonlinear case, Comput. J., 12 (1969), pp. 63-68.

[61] E. Y. Remes, On approximations in the complex domain, Dokl. Akad. Nauk SSSR, 77 (1951), pp. 965-968 (in Russian).

[62] J. R. RIcE, The Approximation of Functions, Vol. 2, Addison-Wesley, Reading, MA, 1969.

[63] J. R. Rice AND K. H. Usow, The Lawson algorithm and extensions, Math. Comp., 22 (1968), pp. $118-127$.

[64] C. Runge, Zur Theorie der eindeutigen analytischen Functionen, Acta Math., 6 (1885), pp. 229-244.

[65] A. RutTan, A characterization of best complex rational approximants in a fundamental case, Constr. Approx., 1 (1985), pp. 287-296.

[66] E. B. SAFF AND R. S. VARGA, Nonuniqueness of best approximating complex rational functions, Bull. AMS, 83 (1977), pp. 375-377.

[67] E. B. SAFf AND R. S. VARga, On the zeros and poles of Padé approximants to $e^{z}$. III, Numer. Math., 30 (1978), pp. 241-266.

[68] C. SChNeIDer AND W. Werner, Some new aspects of rational interpolation, Math. Comp., 47 (1986), pp. 285-299.

[69] G. SHI, On the nonconvergence of the vector fitting algorithm, IEEE Trans. Circuits Syst. II, 63 (2016), pp. 718-722.

[70] J. SigL, Nonlinear residual minimization by iteratively reweighted least squares, Comput. Optim. Appl., 64 (2016), pp. 755-792.

[71] I. Singer, The Theory of Best Approximation and Functional Analysis, SIAM, Philadelphia, 1974, https://doi.org/10.1137/1.9781611970548.book.

[72] H. StahL, Best uniform rational approximation of $|x|$ on $[-1,1]$, Russian Acad. Sci. Sb. Math., 76 (1993), pp. 461-487.

[73] H. Stahl, The convergence of Padé approximants to functions with branch points, J. Approx. Theory, 91 (1997), pp. 139-204.

[74] G. Szegő, Über eine Eigenschaft der Exponentialreihe, Sitzungsber. Berl. Math. Ges., 23 (1924), pp. 50-64.

[75] P. T. P. TANG, A fast algorithm for linear complex Chebyshev approximations, Math. Comp., 51 (1988), pp. 721-739.

[76] J.-P. Thiran And M.-P. Istace, Optimality and uniqueness conditions in complex rational Chebyshev approximation with examples, Constr. Approx., 9 (1993), pp. 83-103.

[77] L. Tonelli, I polinomi d'approssimazione di Tchebychev, Ann. di Mat. Pura ed Appl., 15 (1908), pp. 47-119.

[78] L. N. Trefethen, Chebyshev Approximation in the Complex Plane, undergraduate thesis, Harvard College, Cambridge, MA, 1977.

[79] L. N. Trefethen, Near-circularity of the error curve in complex Chebyshev approximation, J. Approx. Theory, 31 (1981), pp. 344-367.

[80] L. N. Trefethen, Rational Chebyshev approximation on the unit disk, Numer. Math., 37 (1981), pp. 297-320.

[81] L. N. TRefethen, Approximation Theory and Approximation Practice, extended ed., SIAM, Philadelphia, 2019.

[82] L. N. Trefethen, Rational Approximation of the Fermi-Dirac Function, Chebfun example from http://chebfun.org/examples/approx/FermiDirac.html, 2019.

[83] L. N. Trefethen, Numerical conformal mapping with rational functions, Comput. Methods Funct. Theory, (2020), https://doi.org/10.1007/s40315-020-00325-w.

[84] L. N. Trefethen, J. A. C. Weideman, And T. Schmelzer, Talbot quadratures and rational approximations, BIT Numer. Math., 46 (2006), pp. 653-670.

[85] J. Van Deun and L. N. Trefethen, A robust implementation of the Carathéodory-Fejér method for rational approximation, BIT Numer. Math., 51 (2011), pp. 1039-1050.

[86] J. L. WALSH, The existence of rational functions of best approximation, Trans. Amer. Math. Soc., 33 (1931), pp. 668-689.

[87] J. L. WALSh, Interpolation and Approximation by Rational Functions in the Complex Domain, 5th ed., AMS, Providence, RI, 1969.

[88] G. A. Watson, Approximation Theory and Numerical Methods, Wiley, New York, 1980.

[89] H. Werner, Tschebyscheff-Approximation im Bereich der rationalen Funktionen bei Vorliegen einer guten Ausgangsnäherung, Arch. Rational Mech. Anal., 10 (1962), pp. 205-219.

Copyright (c) by SIAM. Unauthorized reproduction of this article is prohibited. 
[90] H. Werner, Die konstruktive Ermittlung der Tschebyscheff-Approximierenden im Bereich der rationalen Funktionen, Arch. Rational Mech. Anal., 11 (1962), pp. 368-384.

[91] W. Werner, Polynomial interpolation: Lagrange versus Newton, Math. Comp., 43 (1984), pp. 205-217.

[92] J. Williams, Characterization and computation of rational Chebyshev approximations in the complex plane, SIAM J. Numer. Anal., 16 (1979), pp. 819-827, https://doi.org/10.1137/ 0716061.

[93] L. Zalcman, Analytic Capacity and Rational Approximation, Lecture Notes in Math. 50, Springer, Berlin, New York, 1968.

Copyright (C) by SIAM. Unauthorized reproduction of this article is prohibited. 